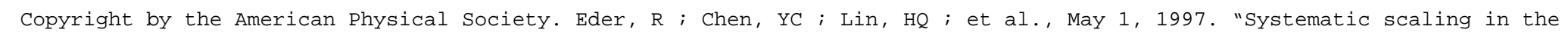

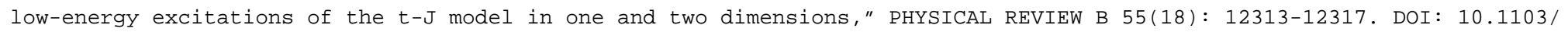

PhysRevB.55.12313.

PHYSICAL REVIEW B

VOLUME 55, NUMBER 18

1 MAY 1997-II

\title{
Systematic scaling in the low-energy excitations of the $t-J$ model in one and two dimensions
}

\author{
R. Eder \\ Department of Applied and Solid State Physics, University of Groningen, Groningen, The Netherlands \\ Y. C. Chen \\ Department of Physics, Tung-Hai University, Taichung, Taiwan \\ H. Q. Lin \\ Department of Physics, Chinese University of Hong Kong, Hong Kong \\ Y. Ohta \\ Department of Physics, Chiba University, Chiba, Japan \\ C. T. Shih \\ Department of Physics, National Tsing Hua University, Hsinchu, Taiwan \\ T. K. Lee \\ Department of Physics, Virginia Tech, Blacksburg, Virginia \\ and Institute of Physics, Academia Sinica, Nankang, Taipei 11529, Taiwan
}

(Received 20 November 1996)

\begin{abstract}
We present an exact diagonalization study of the low-energy singlet and triplet states for both the onedimensional (1D) and 2D $t-J$ models. A scan of the parameter ratio $J / t$ shows that for most low-energy states in both $1 \mathrm{D}$ and $2 \mathrm{D}$ the excitation energy takes the form $E(t, J)=a \cdot t+b \cdot J$. In $1 \mathrm{D}$ this is the natural consequence of the factorization of the low-energy wave functions, i.e., spin-charge separation. Examination of the low-energy eigenstates in 2D shows that most of these are collective modes, which for larger $J$ correspond to a periodic modulation of the hole density. The modulation is well reproduced by treating holes as hard-core bosons with an attractive interaction. [S0163-1829(97)13117-4]
\end{abstract}

Deviations from "generic', Fermi-liquid behavior observed in the normal state of high-temperature superconductors have inspired a search for exotic ground states of strongly correlated electron systems. Thereby a twodimensional (2D) version of the Tomonaga-Luttinger liquid (TLL), which is realized in various 1D systems, ${ }^{1,2}$ has received considerable attention. In a TLL the entire lowenergy excitation spectrum consists of collective excitations, resulting in a "decay" of the physical electron into pairs of such collective modes and consequently rather unusual physics.

Exact diagonalization allows to systematically scan the entire low-energy spectrum of small clusters and even to analyze the wave functions of low-energy states and thus provides a unique tool to obtain approximation-free, unbiased results for 2D strong correlation systems. While the effects of the cluster size pose a serious limitation, it will be shown below that already quite small systems suffice to clearly demonstrate, e.g., the characteristic features of the TLL in 1D. This suggests to seek analogies between rings and planar clusters, and, as will be shown below, 2D systems indeed show some similarity with 1D. In particular, in both cases we find a very regular scaling behavior of the lowest excitation energies as well as evidence for a large number of low-energy collective excitations. The collective charge excitations can be modeled by treating holes as hard-core bosons.
We begin with a discussion of 1D. Figure 1(a) shows the dispersion of the excitation energy $\Delta E$ (i.e., the energy relative to the ground-state energy) for the lowermost singlet and triplet states of a 12-site ring with two holes. Results for both longer and shorter rings (down to six sites) are completely consistent with these. In the $S=0$ sector one can identify two " "branches" of states with very different dependence on $J$ : a first branch (connected by dashed lines) resembles the lower edge of a particle-hole continuum, and has its lowest excitation energy at $2 k_{F}=5 \pi / 6$. The excitation energy roughly scales with $J$, although the energies do not really scale to zero as $J \rightarrow 0$ (which however is likely to be a finite-size effect). A second branch (connected by a full line) comes down at $4 k_{F}=2 \pi / 6$, and is practically invariant under a change of $J$, i.e., its energy scale is $t$. The lowest triplet states closely resemble the dispersion and scaling properties of the singlet $J$ branch, having their minimum excitation energy at $2 k_{F}$. The singlet $t$ branch and triplet $J$ branch have been discussed by Bares and Blatter based on the Betheansatz solution at the supersymmetric point, $J=2 t ;{ }^{2}$ the (near) identity of the low-energy singlet and triplet spectrum is known for the undoped 1D Heisenberg antiferromagnet, where a "singlet replica" of the de Cloiseaux-Pearson mode has been discussed by Mueller et al. ${ }^{3}$ This suggests that in the language of the Ogata-Shiba wave function ${ }^{1}$ the $J$ branches correspond to excitations of the spin part of the wave function, the $t$ branch to an excitation of spinless Fermions or, equivalently, hard-core bosons. 


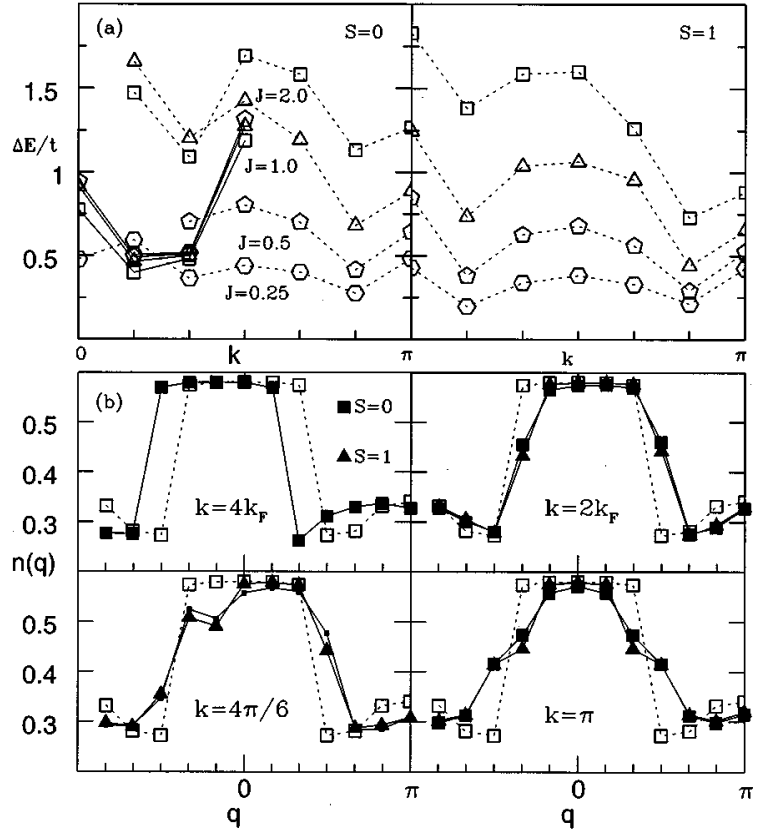

FIG. 1. (a) Lowest excitation energies for two holes in a 12 -site ring for all possible momenta and different $J$. The figure shows the lowest or lowest two states for each momentum, the lines are a guide to the eye. (b) EMD for the ground state of two holes in the 12-site ring (dashed line) compared to $n(q)$ for some excited states with given momentum $k$ (full lines). The ratio $J / t=0.5$.

The unusual nature of the low-energy excitations can be nicely seen in the electron momentum distribution (EMD), defined as $n(q)=\left\langle c_{q, \uparrow}^{\dagger} c_{q, \uparrow}\right\rangle$. This is shown in Fig. 1(b) for some of the low-energy states with $S_{z}=0$. The EMD's of the excited states differ appreciably from that of the ground state only near $\pm k_{F}$, which is, in principle, what one would expect for particle-hole excitations in a Fermi liquid. The only excited states, however, where the change of $n(q)$ would be consistent with the Fermi-liquid picture are the ones with momentum $k=2 k_{F}$. Here the decrease of $n(q)$ at $q=-2 \pi / 6$ and the nearly equal increase at $q=3 \pi / 6$ seem to indicate the shift of an electron between these two momenta, as expected for a particle-hole excitation with a momentum transfer of $k_{F}=5 \pi / 6$. For the excited states with other momenta no such assignment of an electron transfer with the proper momentum is possible (for example the $4 k_{F}$ singlet state "looks like" a particle-hole excitation $2 \pi / 6 \rightarrow-3 \pi / 6$, which however would give a wrong momentum transfer of $-5 \pi / 6$ rather than $2 \pi / 6$ ). These results may be understood by recalling that the low-energy excited states may be thought of ${ }^{2}$ as particle-hole excitations of either a spinon or a holon, whereas an electron near $k_{F}$ corresponds to a combination of low-energy spinon-holon pairs. Changing the occupation numbers of spinons and holons therefore will in turn change the $n(q)$ of the electrons near $k_{F}$. How $n(q)$ changes in detail, however, depends on the (unknown) "expansion", of the physical electrons in terms of spinon-holon pairs, and thus need not at all be consistent with the particle-hole picture.

While the lowermost states correspond to "pure" excitations of either spin or charge degrees of freedom, there is no reason why there should be no mixed excitations, where the spin and charge parts of the wave function are excited simultaneously. This is demonstrated in Fig. 2, which shows the excitation energies, for a larger part of the low-energy spectrum for both two and four holes in the 12-site ring. Some of the excitation energies (indicated by full lines) have the form $\Delta E(t, J)=a \cdot t+b \cdot J$. This could be understood if one were to assume that for these states both, charge and spin degrees of freedom are excited, the former having $t$ as their energy scale, the latter having $J$. When two or more of the linear $\Delta E$ vs $J$ curves intersect, they sometimes produce pronounced hybridization gaps; we believe this is the case, e.g., in the singlet sector for two holes and momentum $5 \pi / 6$. It should be noted that states in one panel are identical in all their possible quantum numbers; nonvanishing matrix elements of the Hamiltonian are therefore possible, and depending on the strength of these matrix elements levels may repel each other appreciably. In addition, there appear to be deviations from the ideal behavior for large $J$; we believe that this is due to the increasing tendency towards hole clustering, which in the thermodynamic limit is known to lead to phase separation for $J$ larger than $J_{c} \approx 3.5 .^{4}$ In the small clusters

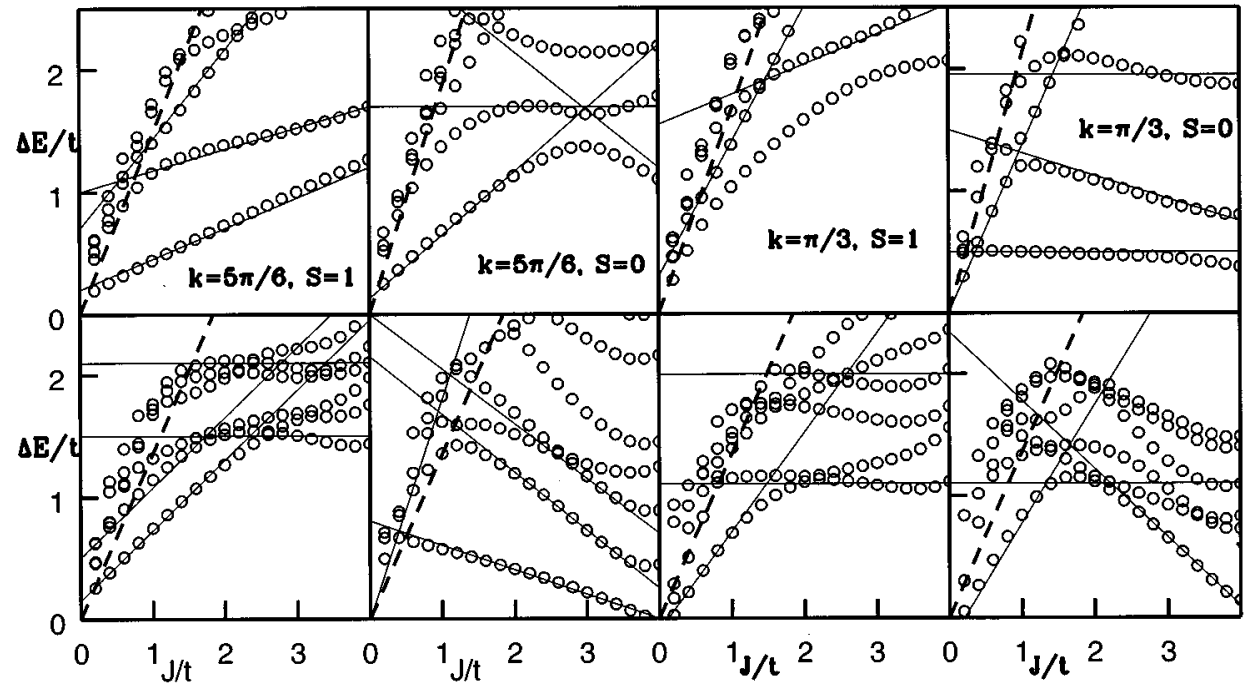

FIG. 2. Lowest excitation energies $\Delta E$ for the 12-site ring with two holes (top row) and four holes (bottom row). Spin and momentum quantum numbers refer to both panels of one column. 


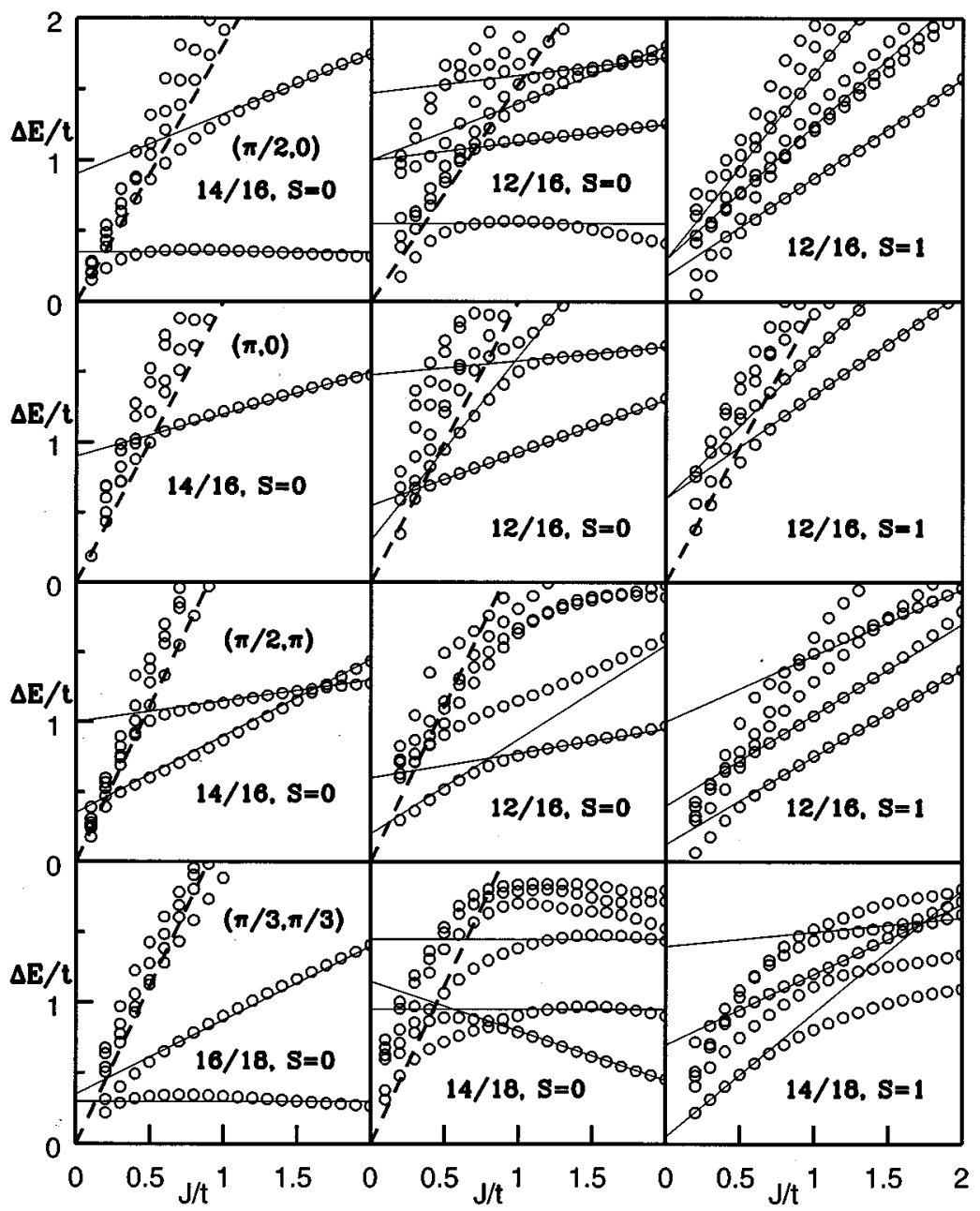

FIG. 3. Lowest excitation energies $\Delta E$ for different 2D cluster sizes $N$ and electron numbers $N_{e}$ in 2D. Each row corresponds to one momentum, the graphs are labeled by electron concentration $N_{e} / N$ and spin $S$. there is no true phase transition, but rather a very slow and gradual crossover to the clustered state, which may lead to a bending of the $\Delta E$ vs $J$ curves. The level crossing at $J / t \approx 4$, where the four-hole singlet state with $k=5 \pi / 6$ comes down below the ground state, most probably is also related to hole clustering. Summarizing the results in $1 \mathrm{D}$, the energies of most of the low-lying eigenstates to a good approximation can be written in the form $\Delta E(t, J)$ $=a \cdot t+b \cdot J$. This can be understood as a consequence of the factorization of the eigenfunctions into a spin part and a spinless fermion part, as established by Ogata and Shiba in the limit $J / t \rightarrow 0$.

Motivated by these systematics we have performed a scan of the $J / t$ dependence of the lowermost excitation energies in $2 \mathrm{D}$. In $2 \mathrm{D}$ point-group symmetry presents a slight complication, in that all allowed momenta for both the 16 and 18 sites clusters are along high-symmetry lines; the respective eigenstates then can be classified according to their parity under reflection by the high-symmetry line. In the following we have only selected those states, whose parity equals that of the ground state, so that they could, in principle, be seen in the dynamical charge-correlation function (DCF). Then, Fig. 3 shows some representative data for the $J$ dependence of the lowest singlet and triplet excitation energies. As was the case for 1D some of the excitation energies (indicated by full lines) have the form $\Delta E(t, J)=a \cdot t+b \cdot J$. As in 1D there are dense continua (indicated by dashed lines) with energy scale
$J$ coming down at small $J$ so that the linear branches "get submerged.' For some of the states the $\Delta E$ vs $J$ curve has a negative curvature; we believe that this is a manifestation of the stronger tendency towards hole clustering in 2D. Extending the range of $J$ up to extreme values such as $J / t=10$, where the energy is essentially determined by the number of broken bonds, the energies of these states approach that of the ground state. We therefore believe that the negative curvature is a consequence of increasing hole clustering, which in the thermodynamic limit leads to phase separation for $J$ greater than $J_{c} \approx>1.5$. $^{5}$ Since this critical value is smaller in $2 \mathrm{D}$ than in $1 \mathrm{D}$ it seems plausible that in $2 \mathrm{D}$ the excitation energies are influenced stronger. Another noteworthy point is that previous studies ${ }^{6}$ indicate that most of the states which contribute significantly in the DCF seem to be "pure $t$ branches.',

Despite some complications the above data show an undeniable analogy between the low-energy spectra of 1D and 2D. To find out more about the nature of the $2 \mathrm{D}$ eigenstates, we proceed to an analysis of their wave functions. We start with a discussion of the static hole density-correlation function, defined as

$$
g(\boldsymbol{R})=\sum_{i}\left\langle\left(1-n_{i}\right)\left(1-n_{i+\boldsymbol{R}}\right)\right\rangle
$$


(a)
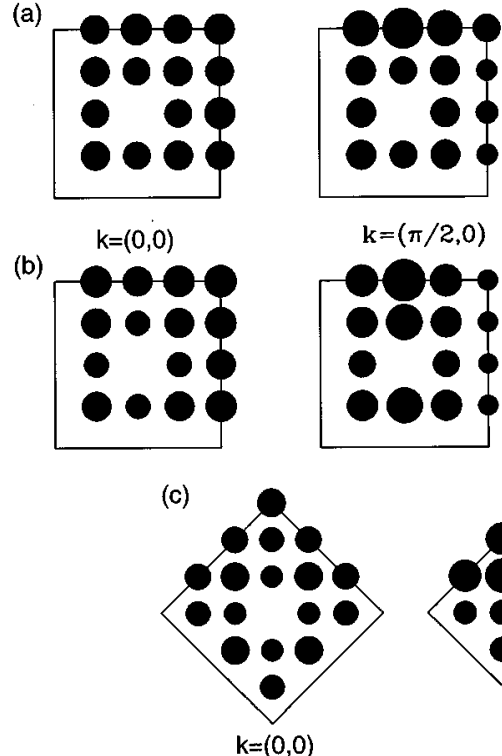

(d)

$k=(0,0)$
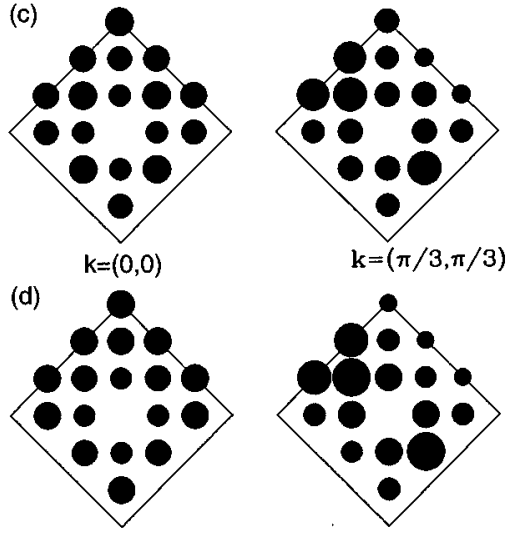

FIG. 4. Hole-density correlation function $g(\boldsymbol{R})$ for the lowest $S=0$ eigenstates with four holes/hard-core bosons and different total momentum $\boldsymbol{k}$ : (a) $t$-J model, 16 sites, (b) hard-core bosons, 16 sites, (c) $t$ - $J$ model, 18 sites, (d) hard-core bosons, 18 sites. The radius of the circle at each $\boldsymbol{R}$ is proportional to the value of $g(\boldsymbol{R})$, the ratio $J / t=1$ for the $t-J$ model and $V / t=1$ for the bosons.

where $n_{i}$ is the operator of electron density at site $i$. The expectation value is taken with the respective eigenstate, some representative results are given in Fig. 4. In the ground state [with momentum $(0,0)]$ for $J / t=1, g(\boldsymbol{R})$ is uniform in 16 sites and even shows a slight decrease at small distances in 18 sites. Contrary to the fairly uniform density correlation in the ground states, the low-energy singlet states show pronounced and systematic modulations of $g(\boldsymbol{R})$. In particular, for momentum along $(1,0)$ these states seem to correspond to "dynamical hole columns." This is reminiscent of the results of Prelovsek and Zotos, ${ }^{7}$ who studied static four-point correlation functions and for large enough $J / t$ found indications of columnlike hole correlations in the ground state. Our scan of the low-energy spectrum indicates that the respective states develop quite continuously down to small values of $J / t$ [for example the state at $\boldsymbol{k}=(\pi, 0)$ has a completely linear $\Delta E$ down to $J=0.5]$; in this physical parameter regime, however, the columnlike structures become nearly unobservable. In previous studies, ${ }^{8,9}$ the correlation of holes was found to be very similar to that of hard-core bosons. By adding an additional nearest-neighbor attractive interaction $V$ between hard-core bosons, we could indeed reproduce the $g(\boldsymbol{R})$ of the excited states of the $t-J$ model surprisingly well, as shown in Fig. 4. We believe that this is an indication that the columns of holes represent a soundlike excitation which gains additional stabilization by a residual short-range attractive interaction between holes.

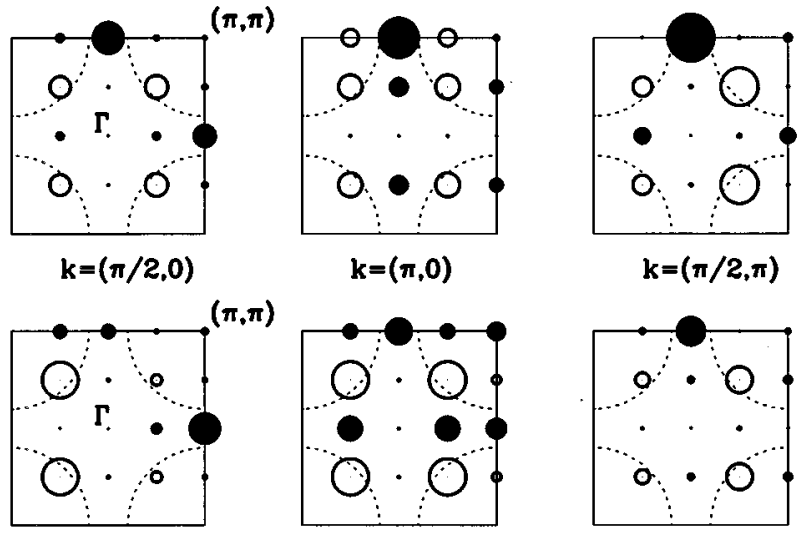

FIG. 5. Difference $\Delta n(\boldsymbol{q})$ for various low-energy states in the $4 \times 4$ cluster with four holes. For each $\boldsymbol{q}$ an open (full) circle indicate a positive (negative) $\Delta n(\boldsymbol{q})$, the radius of the circle is proportional to the magnitude. The ratio $J / t=0.5$, the $\Delta n(\boldsymbol{k})$ refer to the lowest singlet (top row) and triplet (bottom row) state with momentum $\boldsymbol{k}$.

The collective nature of the low-lying states again becomes very obvious in the EMD. More precisely, we consider the difference $\Delta n(\boldsymbol{q})$ of the EMD for an excited state minus the EMD of the ground state; this quantity reflects the redistribution of electrons in momentum space when going over from the ground state to the excited state. Figure 5 shows $\Delta n(\boldsymbol{q})$ for some of the low-energy states of the 16 -site cluster. As was the case in 1D, the changes of $n(\boldsymbol{q})$ occur predominantly in the region of $\boldsymbol{k}$ space where the EMD of the ground state drops sharply; this region is indicated by the dashed line in Fig. 5. Moreover, it is usually impossible to assign transitions of a single electron which could explain the momentum of the state. Let us, for example, consider the $\Delta n(\boldsymbol{q})$ for the triplet state with $\boldsymbol{k}=(\pi / 2,0)$. Here it might seem that an electron has been moved from $(\pi, 0) \rightarrow(-\pi / 2, \pm \pi / 2)$. However, this is inconsistent with the momentum of the state, $(\pi / 2,0)$, so that this state obviously cannot correspond to a single particle-hole excitation. We have performed a similar analysis for other momenta and point-group symmetries, as well as for six holes in 16 sites for both the $t-J$ and Hubbard models,${ }^{12}$ and found consistency with the particle-hole picture only in few cases. The situation is very different at lower electron concentration, where Fermi-liquid like particle-hole transitions can be clearly identified in the EMD for all low-energy states. ${ }^{10}$ Then, summarizing the numerical results for $2 \mathrm{D}$, there is first of all an obvious analogy with 1D. Many of the low-lying excitation energies have a parameter dependence of the form $\Delta E(t, J)=a \cdot t+b \cdot J$, which in $1 \mathrm{D}$ reflects the factorization of the low-lying eigenfunctions. As was the case in 1D most of the low-energy eigenstates in $2 \mathrm{D}$ seem to be collective modes, corresponding to excitations of several different particle-hole pairs. For large enough $J$ some of these develop into "dynamical hole lines." This may partly explain the analogy with 1D, in that a line of holes would cut the spin background into two parts in much the same way as a single hole would do for a 1D system. The lowest of these columnlike states can be followed from the large- $J$ limit down to values as small as $J \approx 0.5-0.3$, and have relatively small 
excitation energies. It should be noted that our clusters allow only for density modulations that are harmonics of the cluster boundary and that finite-size effects most probably tend to increase excitation energies. In an infinite system these collective modes therefore may well appear at low energies and in the physical regime of parameters.

As for the importance of these collective modes for the low-energy physics one could envisage different scenarios. It might be that they simply represent an additional complication "on top of"' a simple Fermi-liquid-like state, i.e., a kind of sound wave. A second possibility would be that, similar as in $1 \mathrm{D}$, the collective modes comprise the entire low-energy spectrum, leading to the well-known "decay" of the physical electron, and thus some 2D analog of the TomonagaLuttinger liquid. Finally, if one were to assume that extremely low energetic hole column excitations persist in some way in the physical parameter range and that these columns' zero-point fluctuations dominate the low-energy physics, one would arrive at the "meandering domain-wall fluid." "However, for any of these scenarios, the fact that the low-energy collective charge excitations also have density correlations similar to hard-core bosons suggest a nonconventional metallic state in this system.

Financial support of R. E. by the European community is most gratefully acknowledged. Part of the research was conducted using the resources of the Cornell Theory Center and National Center for High Performance Computing in Taiwan. This work was partially supported by the National Science Council of Republic of China, Grant Nos. NSC 86-2811-M-007-001R and 86-2112-M-001-042T

\footnotetext{
${ }^{1}$ M. Ogata and H. Shiba, Phys. Rev. B 41, 2326 (1990).

${ }^{2}$ P.A. Bares and G. Blatter, Phys. Rev. Lett. 64, 2567 (1990).

${ }^{3}$ G. Müller et al., Phys. Rev. B 24, 1429 (1981).

${ }^{4}$ M. Ogata et al., Phys. Rev. Lett. 66, 2388 (1991).

${ }^{5}$ W. O. Putikka et al., Phys. Rev. Lett. 68, 538 (1992).

${ }^{6}$ R. Eder et al., Phys. Rev. Lett. 74, 5124 (1995).
}

\footnotetext{
${ }^{7}$ P. Prelovsek and X. Zotos, Phys. Rev. B 47, 5984 (1993).

${ }^{8}$ M.W. Long and X. Zotos, Phys. Rev. B 48, 317 (1993).

${ }^{9}$ Y.C. Chen and T.K. Lee, Z. Phys. B 95, 5 (1994).

${ }^{10}$ R. Eder and Y. Ohta, Phys. Rev. B 51, 11683 (1995).

${ }^{11}$ J. Zaanen et al., Phys. Rev. B 53, 8671 (1996).

${ }^{12}$ Y.C. Chen et al. (unpublished).
} 